\title{
28 Research Square \\ Urgent Care Study of the LumiraDx SARS-CoV-2 Ag Test for Rapid Diagnosis of COVID-19
}

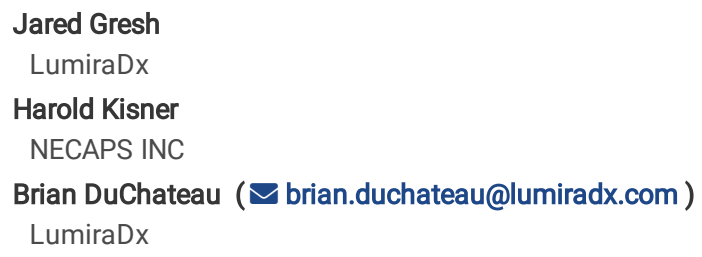

\section{Research Article}

Keywords: antigen testing, community-based setting, COVID-19, false negative, SARS-CoV-2, rapid diagnosis

Posted Date: September 28th, 2021

DOI: https://doi.org/10.21203/rs.3.rs-769498/v2

License: @ (i) This work is licensed under a Creative Commons Attribution 4.0 International License. Read Full License

Version of Record: A version of this preprint was published at Diagnostic and Prognostic Research on December 1st, 2021. See the published version at https://doi.org/10.1186/s41512-021-00113-7. 


\section{Abstract}

Background

Testing individuals suspected of SARS-CoV-2 infection is essential to reduce the spread of disease. The purpose of this study was to determine the false negativity rate of the LumiraDx SARS-CoV-2 Ag Test when utilized for testing individuals suspected of SARS-CoV-2 infection within 12 days of symptom onset.

Methods

Concurrent swab samples were collected from patients suspected of SARS-CoV-2 infection by their healthcare provider within two different urgent care centers. One swab was tested using the LumiraDx SARS-CoV-2 Ag Test. Negative results in patients considered at moderate to high risk of SARS-CoV-2 infection were confirmed at a regional reference laboratory by PCR using the additional swab sample.

Results

From October 19, 2020 - January 3, 2021, a total of 2241 tests were performed using the LumiraDx SARS-CoV-2 Ag Test, with 549 (24.5\%) testing positive and 1692 (75.5\%) testing negative. A subset (800) of the samples rendering a negative LumiraDx SARS-CoV-2 Ag Test were also tested using a PCR-based test for SARS-CoV-2. Of this subset, 770 (96.3\%) tested negative, and 30 (3.8\%) tested positive. A cycle threshold $\left(C_{T}\right)$ was available for 17 of the 30 specimens that yielded discordant results, with an average $C_{T}$ value of 31.2 , an SD of 3.0, and a range of 25.2-36.3. $C_{T}$ was $>30.0$ in $11 / 17$ specimens (64.7\%).

Conclusions

This study demonstrates that negative results obtained with the LumiraDx SARS-CoV-2 Ag Test had $96.3 \%$ agreement with PCR-based SARS-CoV-2 tests, with a low false negative rate of $3.8 \%$ when used in a community-based setting.

\section{Background}

In December, 2019 bronchoalveolar lavage specimens were collected from patients with pneumonia of unknown etiology in Wuhan, China (1). Specimens were shown to be positive for the presence of a beta-coronavirus (1). Whole genome sequencing analysis demonstrated that the viral isolates from 104 different patients showed a sequence homology of $99.9 \%$, suggesting a common disease etiology (1). Full-length genome sequencing showed that the novel viral isolate had a $96 \%$ sequence homology to a bat severe acute respiratory syndrome (SARS)-like coronavirus (CoV) strain called BatCoV RaTG13 (1). The novel virus was named SARS coronavirus 2 (SARS-CoV-2), and the associated disease was named coronavirus disease 2019 (COVID-19) (2). By March 2020 cases of COVID-19 had been identified in over 100 countries, and on March 11, 2020 the World Health Organization (WHO) declared a pandemic (3).

During the first two quarters of 2020, numerous SARS-CoV-2 diagnostic products entered the US market through the US Food and Drug Administration (FDA) emergency use authorization (EUA) program. As of June 2021, 28 different EUA-labeled rapid tests that identify the presence of SARS-CoV-2 antigen are available on the US market, most of which are performed at the point of care with results being available within 30 minutes (4). In contrast, conventional molecular PCR-based methods that are performed in the central diagnostic testing laboratory often take several hours to generate a result and $24-48$ hours to provide a result back to the patient. In many use-cases, rapid SARS-CoV-2 antigen tests can provide a result before the patient even leaves the medical institution at which the test has been administered. The sooner infected individuals are identified, the sooner quarantine and contact tracing can be initiated. Thus, providing a rapid result to identify SARS-CoV-2-infected individuals can help to prevent and truncate the spread of disease (5-7).

Most available rapid SARS-CoV-2 antigen tests use lateral flow technology that has been shown to lack sensitivity compared with PCR (8-11). The rendering of a false negative result to an individual delays or results in the failure to initiate quarantine and contact tracing. As a consequence of a false negative result, many individuals with mild disease may believe they are not infected and may never return for another test, and thus may contribute to the further transmission of disease throughout their disease course.

The LumiraDx SARS-CoV-2 Ag Test (LumiraDx UK Ltd; Alloa, UK) is a rapid microfluidic immunoassay that initially received EUA in August 2020 and identifies the presence of the SARS-CoV-2 nucleocapsid antigen in nasal swabs collected from individuals suspected of COVID-19 by their healthcare provided within 12 days of symptom onset. The test is easy to use, rendering a qualitative, digitally displayed positive or negative result within 12 minutes (12, 13). As high viral loads are known to be detected by antigen tests, this study aimed to focus on the antigen-negative results to determine the percentage of SARS-CoV-2-positive patients that are missed and subsequently confirmed by PCR, in order to determine the impact of introducing the test into a community-based healthcare setting. In this retrospective study, we determined the false negativity rate of the rapid SARS-CoV-2 antigen test from LumiraDx in a cohort of 800 patients within a community-based healthcare setting, by comparing the LumiraDx antigen result with PCR.

\section{Methods}

\section{SARS-CoV-2 patient testing protocol}

All symptomatic patients presenting within 12 days of symptom onset were tested with the LumiraDx SARS-CoV-2 Ag Test. A symptomatic patient was defined as an individual having at least one sign or symptom from those listed in Table 1.

Table 1. Common signs and symptoms of COVID-19 (29). 


\begin{tabular}{|l|}
\hline Common signs and symptoms of COVID-19 \\
Fever or chills \\
Shough \\
Fatigue \\
Muscle or body aches \\
Headache \\
New loss of taste or smell \\
Sore throat \\
Congestion or runny nose \\
Nausea or vomiting \\
Diarrhea
\end{tabular}

COVID-19, coronavirus disease 2019

A subset of patients testing negative with the LumiraDx SARS-CoV-2 Ag Test in routine clinical practice were also tested with a PCR-based test for SARS-CoV2. That subset of patients was determined during the initial clinical and risk assessment. If the patient had a moderate to high likelihood of SARS-CoV-2 infection, then two swabs were concurrently collected. One swab was placed into each nostril concurrently. An anterior nares swab specimen was collected as directed in the manufacturer's package insert. The swabs were then switched and the same swabs were used to collect a specimen from the other nostril. In this way, both of the concurrently collected swabs were equivalent. One swab was then immediately extracted and tested using the LumiraDx SARS-CoV-2 Ag Test (as further described below). If the LumiraDx SARS-CoV-2 Ag Test was positive, the second swab was discarded. If the LumiraDx SARS-CoV-2 Ag Test was negative, the second swab was prepared and sent to a reference laboratory for PCR-based SARS-CoV-2 testing.

\section{Specimen collection and preparation}

The LumiraDx SARS-CoV-2 Ag Test procedure involved the use of a single swab (Puritan HydraFlock Sterile Standard Flock Swab [Puritan Medical Products Company LLC; Guilford, ME, USA], or SteriPack Sterile Polyester Spun Swab [SteriPack USA Ltd LLC; Lakeland, FL, USA]) to swab the anterior nares of both nostrils. Within 1 hour of collection, the nasal swab specimen was placed into the provided vial containing extraction buffer and eluted for 10 seconds. The swab was then swirled along the inside walls of the extraction vial five times. The outside walls of the extraction vial were gently squeezed together as the swab was removed from the vial and discarded. The squeezing action was intended to facilitate the extraction of liquid from the swab. A provided dropper top was then affixed to the top of the extraction vial and the vial was then gently inverted five times. Specimens were interrogated within 5 hours of swab extraction.

\section{SARS-CoV-2 antigen testing}

All SARS-CoV-2 antigen testing was conducted at two urgent care centers within the Compass Medical system. Compass Medical is a physician-owned and directed medical organization providing care to patients of all ages at six different locations across southeastern Massachusetts, USA. Compass Medical is an affiliate of Steward Health Care System, the largest community care organization in New England, USA.

Specimen collection was conducted by a trained clinician using universal precautions and in accordance with the manufacturer's instructions. The LumiraDx SARS-CoV-2 Ag Test is a single-use, rapid microfluidic immunofluorescence assay that is authorized for use under the FDA EUA. The assays intended use states that the test detects the presence of nucleocapsid protein antigen to SARS-CoV-2 directly from nasal swab samples collected from individuals suspected of COVID-19 within the first 12 days of symptom onset. The testing procedure begins by powering on the LumiraDx Instrument. The instrument will prompt to install the lot calibration file when inserting a new test strip lot. Once installed, the instrument will have all the information required to process the test, and any future tests from the same lot of test strips. The instrument will indicate on the touchscreen when to open the foil pouch containing a single reagent test strip. When indicated, the LumiraDx Test Strip is inserted into the LumiraDx Instrument. The instrument will indicate when it is ready for the sample to be applied. When indicated, a single drop from the extracted sample contained within the extraction vial is applied onto the test strip. This is done by gently pressing the sides of the extraction vial until one whole drop of liquid is visible and then allowing it to touch the sample application area indicated on the end of the inserted test strip. The affixed dropper top is calibrated to deliver the required sample volume. The sample is then drawn by capillary action into the test strip. When the sample is detected, the instrument will sound (if sounds are enabled) and a confirmation message will be displayed. The touchscreen of the LumiraDx Instrument will then request the user to immediately close the door. The test result is determined from the amount of fluorescence the instrument detects within the measurement zone of the test strip. The concentration of SARS-CoV-2 antigen in the specimen is proportional to the fluorescence detected. A qualitative result of either POSITIVE or NEGATIVE is displayed on the instrument touchscreen within 12 minutes from applying the sample to the test strip and starting the test.

\section{SARS-CoV-2 PCR-based testing}


PCR-based testing for SARS-CoV-2 was performed at a national reference laboratory (Quest Diagnostics; Marlborough, MA, USA). Nasal swab specimens were collected as described above and in accordance with the recommendations provided in the online test catalogue. The reference laboratory utilized one of three PCR-based methods to conduct SARS-CoV-2 testing. Each of these methods targets different SARS-CoV-2 gene and nucleic acid sequences. Roche, Hologic, and a Quest Diagnostics LDT target SARS-CoV-2 ORF-1a and E, ORF-1, and N1 and N3, respectively. CTs were obtained for all SARS-CoV-2 PCR-based testing performed using the Roche and LDT methods. CTs were not available for SARS-CoV-2 testing performed using the Hologic method. In this study, the average CT between different SARS-CoV-2 gene and nucleic acid sequence targets is reported for each patient specimen represented in Figure 2.

\section{Ethical approval}

This research was performed in accordance with Good Clinical Practice guidelines and the Declaration of Helsinki. Local ethical approval was obtained from Compass Medical Executive Board.

\section{Results}

From October 19, 2020 - January 3, 2021, all symptomatic patients presenting within 12 days of symptom onset, at two different urgent care centers, within a community medical center setting were tested with the LumiraDx SARS-CoV-2 Ag Test. During this time, a total of 2241 patients were tested, with 549 (24.5\%) patients rendering a positive antigen result and 1692 (75.5\%) rendering a negative antigen result (Table 2).

\section{Table 2. SARS-CoV-2 test results from LumiraDx SARS-CoV-2 Ag Test and PCR-based test.}

\begin{tabular}{|ll|}
\hline SARS CoV-2 rapid antigen tests & \\
\hline Total antigen tests conducted* & 2241 \\
Positive antigen results rendered & $549(24.5 \%)$ \\
Negative antigen results rendered & $1692(75.5 \%)$ \\
\hline PCR-based SARS CoV-2 tests & \\
Total PCR-based tests conducted ${ }^{\#}$ & 800 \\
Positive PCR results rendered & $30(3.8 \%)$ \\
Negative PCR results rendered & $770(96.3 \%)$ \\
\hline
\end{tabular}

*All SARS-CoV-2 antigen testing ( $n=2241)$ was conducted using the rapid, point-of-care LumiraDx SARS-CoV-2 Ag Test on patients presenting to an urgent care center.

\#During the initial clinical and risk assessment a subset of patients $(n=800)$ that tested negative with the LumiraDx SARS-CoV-2 Ag Test were also tested with a PCR-based method for SARS-CoV-2.

SARS-CoV-2, severe acute respiratory syndrome coronavirus 2.

During the initial clinical and risk assessment of the patients, 800 (47.3\%) of the patients rendering a negative LumiraDx SARS-CoV-2 Ag Test result were determined to have moderate to high likelihood of SARS-CoV-2 infection, and thus were also tested using a PCR-based test for SARS-CoV-2 (Table 2). This cohort of patients comprised $337(42.1 \%)$ and 463 (57.9\%) males and females respectively, and patients were distributed across six different age categories (Table 3).

Table 3. Age and gender of patients tested with LumiraDx SARS-CoV-2 Ag Test and a PCR-based method.

\begin{tabular}{|llllllll|}
\hline Gender & \multicolumn{6}{l}{ Patient age, $\mathbf{n}(\%)$} \\
\cline { 2 - 8 } & $\mathbf{0 - 1 7}$ & $\mathbf{1 8 - 2 9}$ & $\mathbf{3 0 - 3 9}$ & $\mathbf{4 0 - 4 9}$ & $\mathbf{5 0 - 5 9}$ & $\mathbf{6 0}$ & Total \\
Male & $18(5.3)$ & $98(29.1)$ & $56(16.6)$ & $43(12.8)$ & $58(17.2)$ & $64(19.0)$ & $337(100)$ \\
Female & $16(3.5)$ & $34(7.3)$ & $84(18.1)$ & $117(25.3)$ & $118(25.5)$ & $64(20.3)$ & $463(100)$ \\
& & & & & & $\mathbf{8 0 0}$ \\
\hline
\end{tabular}

PCR, polymerase chain reaction; SARS-CoV-2, severe acute respiratory syndrome coronavirus 2. 
The distribution of this patient cohort in relationship to number of days after symptom onset showed that 732 (91.5\%) of the patients were tested within 7 days after symptom onset, while 40 (5.0\%) were tested more than 7 days after symptom onset (Figure 1). The number of days after symptom onset was not known for three patients, and 25 patients were asymptomatic at the time of testing but had a documented recent exposure to a person testing positive for SARS-CoV-2 (Figure 1). Overall, 770 (96.3\%) of these patients tested negative and 30 (3.8\%) tested positive using a PCR-based test for SARS-CoV-2 (Table 2). This data demonstrates that negative results obtained with the LumiraDx SARS-CoV-2 Ag Test had 96.3\% concordance with PCR-based SARS-CoV-2 tests and a $3.8 \%$ false negativity rate (Table 2 ).

We further investigated the 30 specimens that rendered a negative LumiraDx SARS-CoV-2 Ag Test result and a positive PCR-based test result. Cycle thresholds $\left(C_{T} s\right)$ were available for 17 of 30 (56.8\%) of the specimens tested with a PCR-based testing method (Roche [Roche Cobas 6800 Platform, Roche Molecular Diagnostics, Indianapolis, IN, USA] and a laboratory-developed test [LDT]). $C_{T}$ s were not available for testing performed using the Aptima SARS-CoV-2 Assay (Hologic, Marlborough, MA, USA). The Roche and LDT PCR-based methods target different SARS-CoV-2 genes and nucleic acid sequences. The Roche method targets SARS-CoV-2 ORF-1a and E, while the LDT targets SARS-CoV-2 N1 and N3. In this analysis, the $C_{T} S$ for each target were averaged and reported for each patient, as represented in Figure 2. The $C_{T}$ average for all available $C_{T}$ was $31.2(\mathrm{n}=17)$, with a standard deviation (SD) of 3.0 and a range of $25.2-36.3$. The majority $(64.7 \%)$ of patients testing negative with the LumiraDx SARS-CoV-2 Ag Test and testing positive with a PCR-based test for SARS-CoV-2 had $C_{T} S>30.0$ (Figure 2).

\section{Discussion}

SARS-CoV-2 antigen tests have significant advantages over real-time reverse transcription PCR (RT-PCR) and other nucleic acid amplification tests. Rapid SARS-CoV-2 antigen tests are comparatively inexpensive, can be performed at the point of care, and can provide a result within 30 minutes. Providing a rapid result to identify SARS-CoV-2-infected individuals can help to prevent and truncate the spread of disease, as quarantine and contract-tracing measures can be initiated sooner than in individuals who do not receive such a test (5-7). However, many of the available rapid SARS-CoV-2 antigen tests have been shown to lack sensitivity (8-11). False negative results can have significant public health consequences. In the context of a false negative result, many - individuals with mild disease may believe they are not infected and may never return for another test, and thus may contribute to the further transmission of disease throughout their disease course. In this study we show that the LumiraDx SARS-CoV-2 Ag Test has a low false negativity rate of $3.8 \%$ compared to PCR when used in a community-based healthcare setting.

The product package inserts for other leading rapid SARS-CoV-2 antigen tests report false negative rates similar or even better than those reported for the LumiraDx SARS-CoV-2 Ag Test (14-16). For example, the BinaxNOW COVID-19 Ag Card (Abbott Diagnostics Scarborough, Inc.; Scarborough, ME, USA) and the Veritor System for Rapid Detection of SARS-CoV-2 (Becton, Dickinson and Company; Sparks, MD, USA) report false negative rates of 5.1\% and 2.5\% respectively, while the Sofia 2 Flu + SARS Antigen FIA (Quidel Corporation; San Diego, CA, USA) reports a false negative rate of only 0.6\% (14-16). However, the false negativity rate is influenced by disease prevalence and hence false negativity rates may not be directly comparable, especially when disease prevalence varies significantly. In fact, the false negativity rates for these products were much higher when deployed and determined in an actual community setting $(11,17-19)$. Furthermore, there are significant differences in the studies represented in the product package inserts compared with the study reported here. The studies represented in the product package inserts are all smaller than the study of 800 patients reported here, with the BinaxNOW COVID-19 Ag Card, Veritor System for Rapid Detection of SARS-CoV-2, and Sofia 2 Flu + SARS Antigen FIA studies consisting of 460, 226, and 164 patients respectively (1416).Furthermore, the study of the Veritor System for Rapid Detection of SARS-CoV-2 only included symptomatic patients within 5 days of symptom onset (15). The studies of the BinaxNOW COVID-19 Ag Card and Sofia 2 Flu + SARS Antigen FIA included symptomatic patients within 7 days of symptom onset, but the majority of patients included in the Sofia 2 Flu + SARS Antigen FIA study were within 4 days after symptom onset $(14,16)$. The study reported here included symptomatic patients within up to 12 days of symptom onset.

A false negative test result could be rendered when SARS-CoV-2 viral loads are low, such as very early $(20,21)$ and very late (22) in the disease course of COVID-19. Studies have demonstrated that rapid SARS-CoV-2 antigen tests have the greatest sensitivities when viral loads are high (23, 24). This suggests that the false negativity rate of the BinaxNOW COVID-19 Ag Card, the Veritor System for Rapid Detection of SARS-CoV-2, and the Sofia 2 Flu + SARS Antigen FIA could have been higher if the respective studies had included patients who were tested more than 7 days of symptom onset, when viral load levels would have been declining. In support of this hypothesis, studies have demonstrated that the limit of detection for the Veritor System for Rapid Detection of SARSCoV-2 and the Sofia 2 Flu + SARS Antigen FIA has a sensitivity equivalent to a molecular $C_{T}$ of 27-28 (25). In contrast, the LumiraDx SARS-CoV-2 Ag Test is more sensitive, with a sensitivity equivalent to a molecular $C_{T}$ of $<33$ (13). The increased sensitivity of the LumiraDx SARS-CoV-2 Ag Test versus other rapid antigen tests is likely the consequence of the testing methodology. The LumiraDx SARS-CoV-2 Ag Test is a rapid microfluidic immunofluorescence assay that utilizes active control of the reaction time, volume, and temperature. The assay utilizes a wash step and a spectrophotometric read. In contrast, the BinaxNOW COVID-19 Ag Card, Veritor System for Rapid Detection of SARS-CoV-2, and Sofia 2 Flu + SARS Antigen FIA all utilize conventional lateral flow testing methodology. Lateral flow test methods entail only passive control of the specimen, with no wash steps.

The BinaxNOW COVID-19 Ag Card and the Sofia 2 Flu + SARS Antigen FIA both have FDA EUA claims for utilization in patients within up to 7 days of symptom onset, while the Veritor System for Rapid Detection of SARS-CoV-2 has an FDA EUA claim for utilization in patients within up to 5 days of symptom onset. Studies have demonstrated that SARS-CoV-2 can be successfully cultured in symptomatic patients 8-15 days following symptom onset (26-

28). If the presence of culturable virus provides at least some measure of infectivity, then it would be important that a rapid SARS-CoV-2 antigen test be able to identify infected patients more than 7 days after symptom onset. The LumiraDx SARS-CoV-2 Ag Test has an FDA EUA claim for utilization in patients up to 12 
days after symptom onset, and this study demonstrated that the LumiraDx SARS-CoV-2 Ag Test has a low false negative rate of $3.8 \%$ compared to PCR, even when patients presenting up to 12 days after symptom onset were included in the analysis.

This study also demonstrated that $11 / 17$ (64.7\%) of the false negative results rendered by the LumiraDx SARS-CoV-2 Ag Test had $C_{T} s>30$. This suggests that many of the specimens rendering a false negative result with the LumiraDx SARS-CoV-2 Ag Test had low viral loads and were potentially non-infectious (13). A previous study demonstrated that the LumiraDx SARS-CoV-2 Ag Test had 100\% concordance with SARS-CoV-2 PCR-positive specimens with $C_{T} s<33$ (13). The difference between the results of this previous study and those reported here may be reflective of the differences in the PCR assays used in each study. Drain et al. utilized the Roche SARS-CoV-2 PCR assay, while the study presented here primarily utilized an LDT SARS-CoV-2 PCR assay (13). $C_{T} S$ achieved across different PCR-based assays are not directly comparable.

\section{Conclusions}

Additional studies are needed to better characterize when individuals with SARS-CoV-2 infection are infectious and thus capable of transmitting disease. The development and optimization of the clinical and analytic performance of diagnostic tests to identify infectious individuals is of the utmost importance to public health. This study demonstrated a low false negative rate when using the LumiraDx SARS-CoV-2 Ag Test in a community-based setting.

\section{Abbreviations}

$\mathrm{Ag}$

antigen

COVID-19

coronavirus disease 2019

CT

cycle threshold

EUA

US Food and Drug Administration (FDA) emergency use authorization (EUA) program

FDA

US Food and Drug Administration

LDT

lab developed test

ORF

open reading frame

PCR

polymerase chain reaction

RT-PCR

real-time reverse transcription $\mathrm{PCR}$

SARS-CoV-2

severe acute respiratory syndrome (SARS)-like coronavirus (CoV) 2

SD

standard deviation

WHO

World Health Organization

\section{Declarations}

\section{Ethics approval and consent to participate}

This research was performed in accordance with Good Clinical Practice guidelines and the Declaration of Helsinki. Local ethical approval was obtained from Compass Medical Executive Board.

The compass testing protocol was formulated independent of the study based on recommendations from the Compass Medical Chief Medical Officer and Urgent Care Physicians. The study represents a retrospective analysis of routine clinical practice. No additional specimens were collected in support of the study and thus consent was not collected nor needed.

\section{Consent for publication}

No individual patient data has been included in this article.

\section{Availability of data and materials}


The datasets analyzed during the current study are available from the corresponding author on reasonable request.

\section{Competing interests}

Jared Gresh and Brian DuChateau are employees of LumiraDx, USA. Harold Kisner is a LumiraDx consultant and stockholder.

\section{Funding}

The study was funded by LumiraDx. The funders had no role in the study design or data collection.

\section{Authors' contribution}

JG made substantial contributions to the acquisition, analysis, and interpretation of data; HK made substantial contributions to the interpretation of data and critical review of the manuscript; BDuC made substantial contributions to the data analysis, interpretation of data, and drafted the manuscript. All authors approved the submitted version and agreed both to be personally accountable for the author's own contributions and to ensure that questions related to the accuracy or integrity of any part of the work, even ones in which the author was not personally involved, are appropriately investigated, resolved, and the resolution documented in the literature. All authors have read and approved the final manuscript.

\section{Acknowledgements}

The authors acknowledge Viola Kooij, PhD, of integrated medhealth communication (imc), UK, for editorial support, which was funded by LumiraDx UK Ltd.

\section{References}

1. World Health Organization. Report of the WHO-China Joint Mission on Coronavirus Disease 2019 (COVID-19). Available from: https://www.who.int/docs/default-source/coronaviruse/who-china-joint-mission-on-covid-19-final-report.pdf (Accessed April 2021).

2. Coronaviridae Study Group of the International Committee on Taxonomy of Viruses. The species severe acute respiratory syndrome-related coronavirus: classifying 2019-nCoV and naming it SARS-CoV-2. Nat Microbiol. 2020;5:536-44.

3. World Health Organisation. WHO Director-General's opening remarks at the media briefing on COVID-19-11 March 2020. Available from: https://www.who.int/director-general/speeches/detail/who-director-general-s-opening-remarks-at-the-media-briefing-on-covid-19-11-march-2020 (Accessed June 2021).

4. US Food and Drug Administration. In vitro diagnostics EUAs - antigen diagnostic tests for SARS-CoV-2. Available from: https://www.fda.gov/medicaldevices/emergency-situations-medical-devices/coronavirus-covid-19-and-medical-devices (Accessed June 2021).

5. Fraser C, Riley S, Anderson RM, Ferguson NM. Factors that make an infectious disease outbreak controllable. Proc Natl Acad Sci USA. 2004;101:6146-51.

6. Lipsitch M, Cohen T, Cooper B, Robins JM, Ma S, James L, et al. Transmission dynamics and control of severe acute respiratory syndrome. Science. 2003;300:1966-70.

7. Sanche S, Lin YT, Xu C, Romero-Severson E, Hengartner N, Ke R. High contagiousness and rapid spread of severe acute respiratory syndrome coronavirus 2. Emerg Infect Dis. 2020;26:1470-7.

8. Ferguson J, Dunn S, Best A, Mirza J, Percival B, Mayhew M, et al. Validation testing to determine the effectiveness of lateral flow testing for asymptomatic SARS-CoV-2 detection in low prevalence settings. medRxiv 2020:2020.12.01.20237784.

9. Mak GC, Cheng PK, Lau SS, Wong KK, Lau CS, Lam ET, et al. Evaluation of rapid antigen test for detection of SARS-CoV-2 virus. J Clin Virol. 2020;129:104500.

10. Peto T. COVID-19: Rapid antigen detection for SARS-CoV-2 by lateral flow assay: A national systematic evaluation for mass-testing. medRxiv 2021:2021.01.13.21249563.

11. Pray IW, Ford L, Cole D, Lee C, Bigouette JP, Abedi GR, et al. Performance of an antigen-based test for asymptomatic and symptomatic SARS-CoV-2 testing at two university campuses - Wisconsin, September-October 2020. MMWR Morb Mortal Wkly Rep. 2021;69:1642-7.

12. Krüger LJ, Klein JAF, Tobian F, Gaeddert M, Lainati F, Klemm S, et al. Evaluation of accuracy, exclusivity, limit-of-detection and ease-of-use of LumiraDx ${ }^{\text {TM }}$ Antigen-detecting point-of-care device for SARS-CoV-2. medRxiv 2021:2021.03.02.21252430.

13. Drain PK, Ampajwala M, Chappel C, Gvozden AB, Hoppers M, Wang M, et al. A rapid, high-sensitivity SARS-CoV-2 nucleocapsid immunoassay to aid diagnosis of acute COVID-19 at the point of care: A clinical performance study. Infect Dis Ther 2021:1-9.

14. Abbott. BinaxNOW COVID-19 Ag Card - Instructions for use. Last revised December 2020.. Available from: https://www.fda.gov/media/141570/download (Accessed April 2021).

15. BD. BD Veritor system for rapid detection of SARS-CoV-2 - Instructions for use. Last revised March 2021. Available from: https://www.fda.gov/media/139755/download\#: :text=from\%20respiratory\%20specimens.-,The\%20BD\%20Veritor\%E2\%84\%A2\%20System\%20for\%20Ra (Accessed April 2021).

16. Quidel Corporation. Sofia 2 Flu + SARS antigen FIA - Instructions for use. Available from: https://www.fda.gov/media/142704/download (Accessed April 2021). 
17. Beck ET, Paar W, Fojut L, Serwe J, Jahnke RR. Comparison of the Quidel Sofia SARS FIA Test to the Hologic Aptima SARS-CoV-2 TMA Test for Diagnosis of COVID-19 in Symptomatic Outpatients. J Clin Microbiol. 2021;59:e02727-20.

18. Prince-Guerra JL, Almendares O, Nolen LD, Gunn JKL, Dale AP, Buono SA, et al. Evaluation of Abbott BinaxNOW Rapid Antigen Test for SARS-CoV-2 infection at two community-based testing sites - Pima County, Arizona, November 3-17, 2020. MMWR Morb Mortal Wkly Rep. 2021;70:100-5.

19. Young S, Taylor SN, Cammarata CL, Varnado KG, Roger-Dalbert C, Montano A, et al. Clinical evaluation of BD Veritor SARS-CoV-2 Point-of-Care Test performance compared to PCR-based testing and versus the Sofia 2 SARS Antigen Point-of-Care Test. J Clin Microbiol. 2020;59:e02338-20.

20. He X, Lau EHY, Wu P, Deng X, Wang J, Hao X, et al. Temporal dynamics in viral shedding and transmissibility of COVID-19. Nat Med. 2020;26:672-5.

21. Wolfel R, Corman VM, Guggemos W, Seilmaier M, Zange S, Muller MA, et al. Virological assessment of hospitalized patients with COVID-2019. Nature. 2020;581:465-9.

22. Kucirka LM, Lauer SA, Laeyendecker O, Boon D, Lessler J. Variation in false-negative rate of reverse transcriptase polymerase chain reaction-based SARSCoV-2 tests by time since exposure. Ann Intern Med. 2020;173:262-7.

23. Gremmels H, Winkel BMF, Schuurman R, Rosingh A, Rigter NAM, Rodriguez O, et al. Real-life validation of the Panbio COVID-19 antigen rapid test (Abbott) in community-dwelling subjects with symptoms of potential SARS-CoV-2 infection. EClinicalMedicine. 2021;31:100677.

24. Merino-Amador P, Guinea J, Muñoz-Gallego I, González-Donapetry P, Galán J-C, Antona N, et al. Multicenter evaluation of the Panbio'm COVID-19 Rapid Antigen-Detection Test for the diagnosis of SARS-CoV-2 infection. medRxiv 2020:2020.11.18.20230375.

25. Pollock NR, Savage TJ, Wardell H, Lee R, Mathew A, Stengelin M, et al. Correlation of SARS-CoV-2 nucleocapsid antigen and RNA concentrations in nasopharyngeal samples from children and adults using an ultrasensitive and quantitative antigen assay. medRxiv 2020:2020.11.10.20227371.

26. Bullard J, Dust K, Funk D, Strong JE, Alexander D, Garnett L, et al. Predicting infectious severe acute respiratory syndrome coronavirus 2 from diagnostic samples. Clin Infect Dis. 2020;71:2663-6.

27. Seow J, Graham C, Merrick B, Acors S, Pickering S, Steel KJA, et al. Longitudinal observation and decline of neutralizing antibody responses in the three months following SARS-CoV-2 infection in humans. Nat Microbiol. 2020;5:1598-607.

28. van Kampen JJA, van de Vijver DAMC, Fraaij PLA, Haagmans BL, Lamers MM, Okba N, et al. Duration and key determinants of infectious virus shedding in hospitalized patients with coronavirus disease-2019 (COVID-19). Nat Commun. 2021;12:267.

29. Centers for Disease Control and Prevention. Symptoms of COVID-19. Available from: https://www.cdc.gov/coronavirus/2019-ncov/symptomstesting/symptoms.html (Accessed April 2021).

\section{Figures}

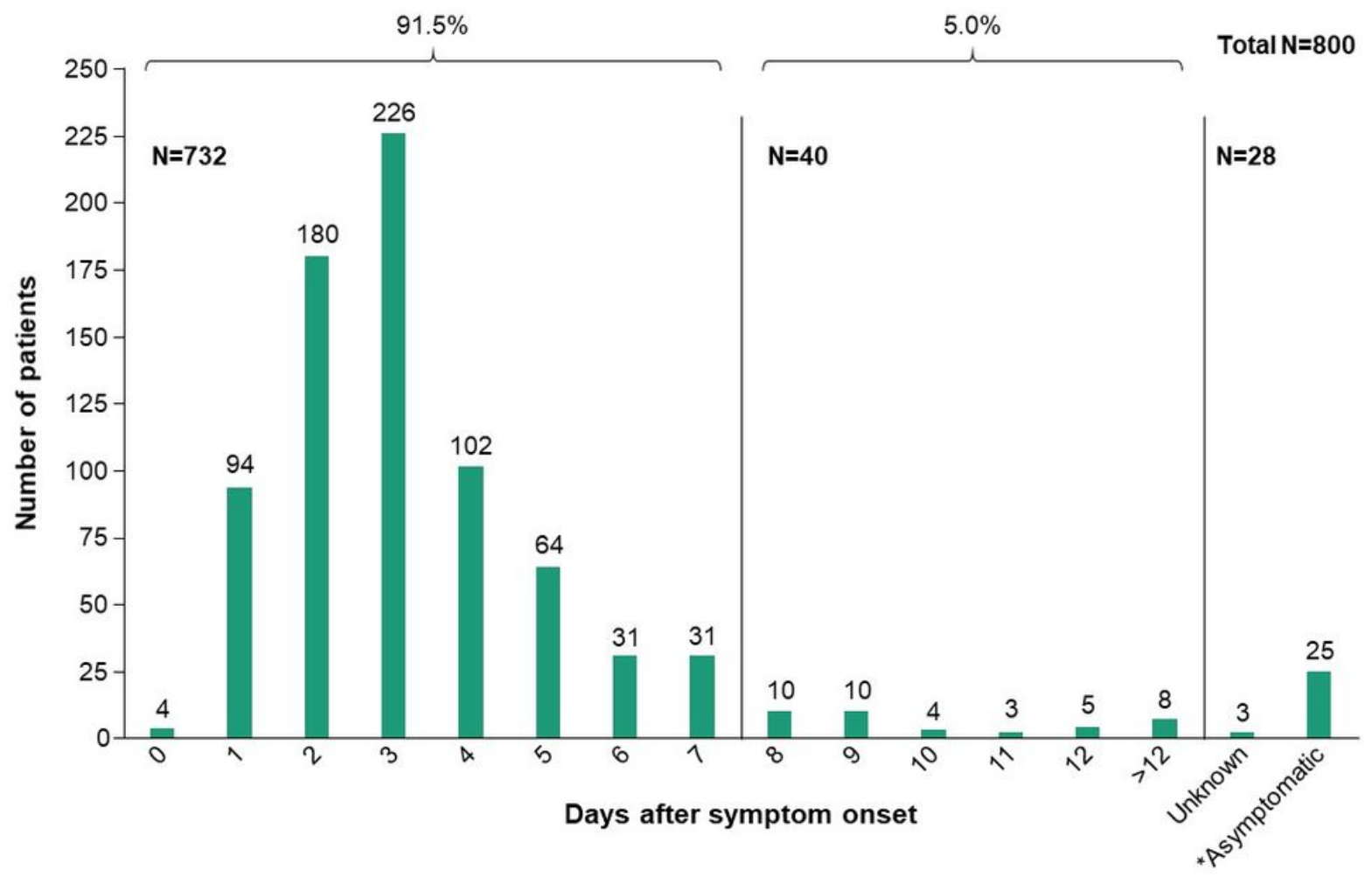

Figure 1 
Days after symptom onset for patients tested with LumiraDx SARS-CoV-2 Ag Test and PCR-based method. There were 91.5\% (732/800) of the patients tested within 7 days of symptom onset, while $5 \%$ (40/800) were tested more than 7 days after symptom onset. Number of days after symptom onset was not known in three patients, and 25* patients were asymptomatic at the time of testing but had a recent documented exposure to a person testing positive for SARS-CoV2. PCR, polymerase chain reaction; SARS-CoV-2, severe acute respiratory syndrome coronavirus 2.

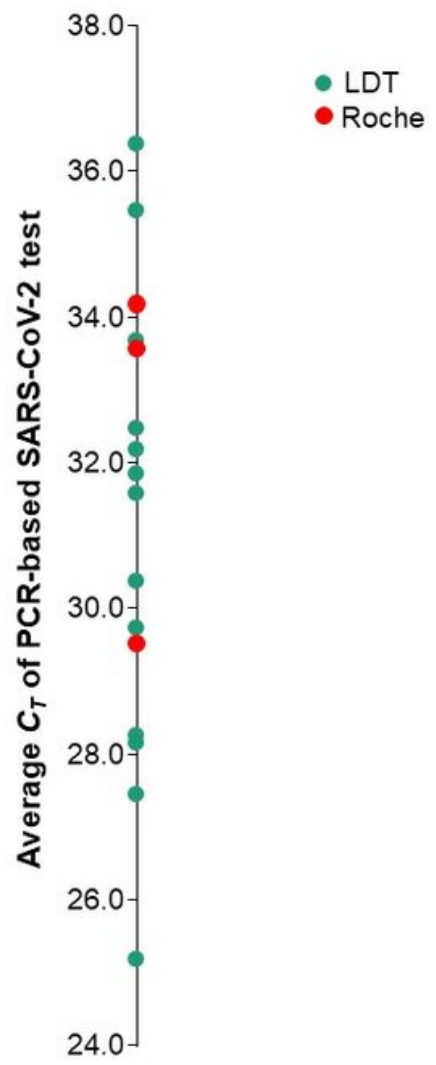

Figure 2

CTs were available for $56.8 \%$ of negative LumiraDx SARS-CoV-2 Ag Tests with positive PCR results. The average CT between different SARS-CoV-2 gene and nucleic acid sequence targets is reported for each patient specimen represented. CTs were not available for SARS-CoV-2 testing performed using the Hologic method. CT, cycle threshold; Green circles, LDT, a laboratory-developed PCR-testing method; PCR, polymerase chain reaction; Red circles, Roche PCR-based testing method; SARS-CoV-2, severe acute respiratory syndrome coronavirus 2. 